Nuclear Instruments and Methods in Physics Research Section A: Accelerators, Spectrometers, Detectors and Associated Equipment

Vol. 567, Issue 2 , 15 Nov. 2006, Pages 527-530

http://dx.doi.org/10.1016/..nima.2006.05.212

(c) 2006 Elsevier B.V. All rights reserved
Archimer, archive institutionnelle de l'Ifremer http://www.ifremer.fr/docelec/

\title{
The deep-sea hub of the ANTARES neutrino telescope
}

\author{
M. Anghinolfi ${ }^{a}$, A. Calzas ${ }^{b}$, B. Dinkespiler ${ }^{b}$, S. Cuneo $^{c}$, S. Favard ${ }^{b}$, G. Hallewell ${ }^{b},{ }^{*}, M^{\circ}$. Jaquet ${ }^{b}$, \\ M. Musumecic, R. Papaleo ${ }^{c}$, G. Raia ${ }^{c}$, P. Valdy ${ }^{d}$ and P. Vernin ${ }^{e}$
}

aINFN Sezione di Genova, Via Dodecaneso 33, I-16146 Genova, Italy

${ }^{\mathrm{b}}$ Centre de Physique des Particules de Marseille (CNRS/IN2P3), Université de la Méditerranée, 13288 Marseille, France

'INFN Laboratori Nazionali del Sud, Via S. Sofia 44, I-95123 Catania, Italy

dIFREMER(Institut français de recherche pour l'exploitation de la mer), Centre de La Seyne, 83500 La Seyne sur mer, France

${ }^{\text {e}}$ DSM-DAPNIA, CEA SACLAY, 91191 Gif sur Yvette Cedex, France

*: Corresponding author : Tel.: +33 4918276 00; fax: +33 4918272 99; gregh@cppm.in2p3.fr

\begin{abstract}
The ANTARES neutrino telescope, currently under construction at $2500 \mathrm{~m}$ depth off the French Mediterranean coast, will contain 12 detection lines, powered and read out through a deep-sea junction box (JB) hub. Electrical energy from the shore station is distributed through a transformer with multiple secondary windings and a plugboard with 16 deep sea-mateable electro-optic connectors. Connections are made to the JB outputs using manned or remotely operated submersible vehicles. The triply redundant power management and slow control system is based on two identical ACpowered systems, communicating with the shore through $160 \mathrm{Mb} / \mathrm{s}$ fibre G-links and a third batterypowered system using a slower link.
\end{abstract}

We describe the power and slow control systems of the underwater hub.

Keywords: Underwater neutrino telescope; Power distribution; Slow control system 


\title{
The deep-sea hub of the ANTARES neutrino telescope
}

\author{
M. Anghinolfi ${ }^{\mathrm{a}}$, A. Calzas ${ }^{\mathrm{b}}$, B. Dinkespiler ${ }^{\mathrm{b}}, \mathrm{S} . \mathrm{Cuneo}^{\mathrm{c}}, \mathrm{S}$. Favard ${ }^{\mathrm{b}}$, G. Hallewell ${ }^{\mathrm{b} *}$, \\ M. Jaquet ${ }^{\mathrm{b}}$, M. Musumeci ${ }^{\mathrm{c}}$, R. Papaleo ${ }^{\mathrm{c}}, \mathrm{G}_{\text {. Raia }}^{\mathrm{c}}, \mathrm{P}$. Valdy ${ }^{\mathrm{d}}$ and P. Vernin ${ }^{\mathrm{e}}$ \\ ${ }^{a}$ INFN Sezione di Genova, Via Dodecaneso 33, I-16146 Genova, Italy \\ ${ }^{b}$ Centre de Physique des Particules de Marseille (CNRS/IN2P3), Université de la Méditerranée, 13288 Marseille, France
}

${ }^{c}$ INFN Laboratori Nazionali del Sud, Via S. Sofia 44, I-95123 Catania, Italy

${ }^{d}$ IFREMER(Institut français de recherche pour l'exploitation de la mer), Centre de La Seyne, 83500 La Seyne sur mer, France

${ }^{e}$ DSM-DAPNIA, CEA SACLAY, 91191 Gif sur Yvette Cedex, France

\begin{abstract}
The ANTARES neutrino telescope, currently under construction at 2500m depth off the French Mediterranean coast, will contain twelve detection lines, powered and read out through a deep-sea junction box hub. Electrical energy from the shore station is distributed through a transformer with multiple secondary windings and a plugboard with sixteen deep sea-mateable electro-optic connectors. Connections are made to the junction box outputs using manned or remotely operated submersible vehicles. The triply-redundant power management and slow control system is based on two identical AC-powered systems, communicating with the shore through $160 \mathrm{Mb} / \mathrm{s}$ fibre G-links and a third battery-powered system using a slower link.

We describe the power and slow control systems of the underwater hub.
\end{abstract}

Keywords: Underwater neutrino telescope; power distribution; slow control system

\section{Introduction}

When complete in 2007, the ANTARES telescope, [1,2,3; Fig.1] will detect Čerenkov light from muons

produced in the interactions of high energy neutrinos using twelve detection lines, each containing 75 photomultipliers housed in glass pressure spheres, organized in 25 'triplets' between 100 and $450 \mathrm{~m}$ from the sea floor. The lines will be anchored on a sixty-metre sea floor grid and tensioned by submerged buoys. Cables will link the base of each line to a junction box (JB) hub through which power is distributed and data collected.

The JB, described in the following sections, is connected to the shore station near Toulon through a $41 \mathrm{~km}$ electro-optical cable and has been in continuous communication with the shore since its deployment in December 2002.

\footnotetext{
* Corresponding author. Tel +33 491827642; fax +33 491827299; e-mail: gregh@cppm.in2p3.fr
} 


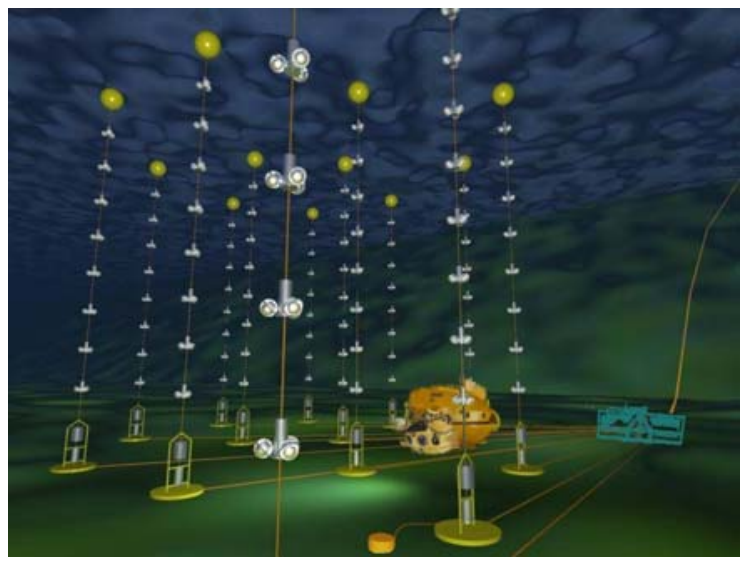

Fig. 1. The ANTARES underwater neutrino telescope.

\section{Junction box mechanics and deployment}

The JB structure (Fig. 2), is based on a pair of $1 \mathrm{~m}$ diameter titanium hemispheres, separated by a central cylinder ("belt") through which all power and data connections pass. The internal pressure is $1 \mathrm{bar}_{\mathrm{abs}}$ while the ambient external sea pressure is 250bar. Each hemisphere is sealed to the belt with two concentric ' $\mathrm{O}$ ' rings. The lower hemisphere contains an oil-immersed 24KVA transformer while the upper hemisphere contains the power system slow control electronics. Following component installation, the JB sphere was pre-qualified in a 24 hour pressure test at 310 bar.

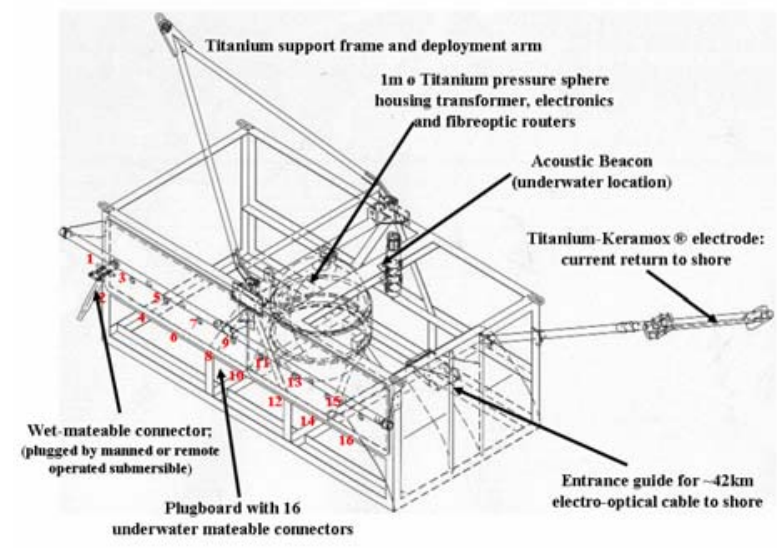

Fig. 2. The junction box sphere and its support cage.
The sphere is supported within a rectangular titanium transit cage suspended from a pivoting arm during deployment. The cage carries a transponder for acoustic triangulation of the frame position during deployment, an electrode for current return to the shore and a plugboard with deep-sea mateable electro-optical connectors allowing cable connections to the 16 outputs using the manipulator arms of a submersible vehicle.

\section{Junction box power system and cabling}

\subsection{Power supply to the junction box}

A shore station located at the cable landing beach raises the voltage from $400 \mathrm{~V} 50 \mathrm{~Hz}$ to the range 3700 $4100 \mathrm{~V}$ for passage through the undersea cable to the JB. The voltage at the cable input is adjusted using a motor driven variable transformer, depending on the load requirement (the number of lines to be powered).

An AC power system [4, 5, Fig. 3] was chosen as the best compromise for power delivery over the $41 \mathrm{~km}$ transmission distance. The phase shift due to the $9 \mu \mathrm{F}$ cable capacitance is largely compensated with a $1.4 \mathrm{H}$ self inductance at the shore end of the cable. Although losses are greater than in a DC system with the same voltage and current limits, AC operation allows passive (transformer) variation of the cable entry voltage and greater reliability at the sea-bed distribution hub through use of a transformer.

The undersea cable ${ }^{1}$ contains 48 monomode optical fibres and a single conductor (normally used for series powering of repeaters in long haul cables) and delivers a current up to a 9A maximum to the $\mathrm{HV}$ pole of the JB transformer primary winding. The LV pole returns current via an external electrode ${ }^{2}$ to the sea and thence to the shore station, which has receiving electrodes buried in the nearby ground. The use of a sea return reduces ohmic losses by a factor 4 compared with a cable sharing equivalent section between supply and return conductors.

\footnotetext{
${ }^{1}$ Alcatel URC3 Type 4 (unrepeatered)

${ }^{2}$ Titanium with Keramox ${ }^{\circledR}$ coating, of length $1.6 \mathrm{~m}$ and diameter 40mm; Mfr Magneto BV 3125 Schiedam, Netherlands
} 


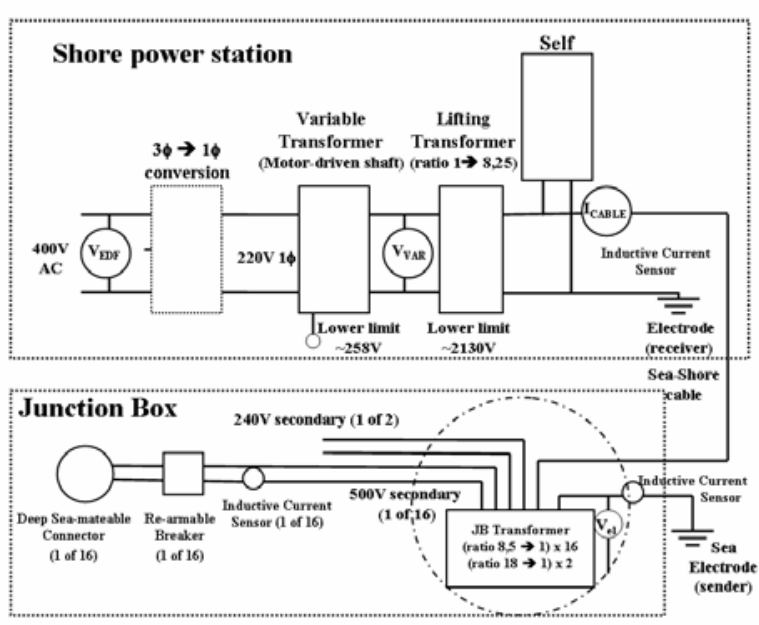

Fig. 3. Power distribution system: shore and deep-sea junction box.

The JB has 16 outputs for connection of detection lines and oceanographic instruments. The typical power draw for a detection line is $\sim 1 \mathrm{~kW}$. The JB outputs are galvanically separated through the use of a transformer with 16 individual 500V-rated secondaries. Two additional $240 \mathrm{~V}$ windings power the JB internal slow control systems. Each output is protected by a thermo-magnetic breaker ${ }^{3}$ set to $5 \mathrm{~A}$ threshold.

Underwater connections are made via oil-filled electro-optical connectors ${ }^{4}$, mateable in seawater (Fig. 4) at ambient pressures exceeding 250bar.

Each connection point has four optical fibres and a pair of electrical conductors providing AC power in the range $435-480 \mathrm{~V}$. The conductors and optical fibres of unmated output connectors are protected from sea water exposure by a shutter, until the final phase of insertion.

Breakers may be rearmed or opened with any of three independent control channels ('A','B'\& 'C') through the wire-OR powering of intermediate $240 \mathrm{~V}$ relays (Fig. 5). Breakers corresponding to unused outputs are kept in the closed (powered) position, both to minimise the number of breaker operations and also to allow early detection of water infiltration past the shutter of a connector, which would be manifested as increased leakage current on a sensitive current sensor. ${ }^{5}$

\footnotetext{
${ }^{3}$ Moller PKZ2/ZN6 with RE-PZK2 remote control block

${ }^{4} \mathrm{Hybrid}$ electro-optical connector with penetrator and $2.5 \mathrm{~m}$ jumper cable (ref 1009533); Ocean Design Inc, Ormond Beach, FL32174,
}

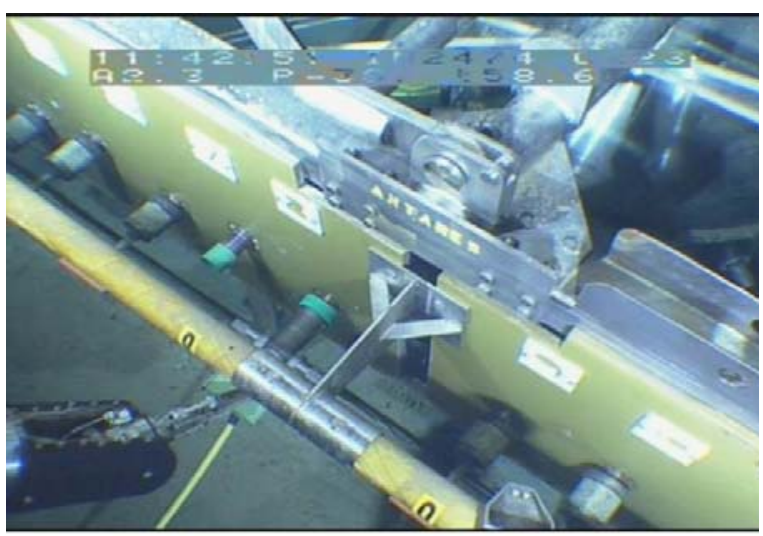

Fig. 4. Umbilical connection to a junction box output connector by the IFREMER Nautile manned submersible (March 2003).

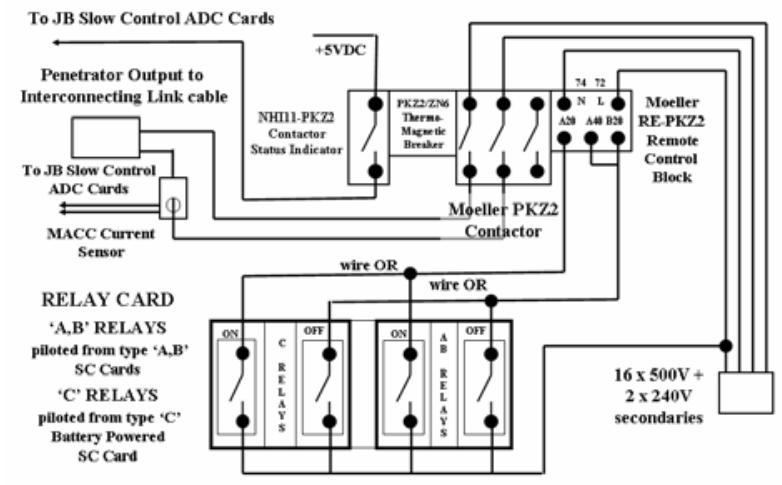

Fig. 5 Junction box output breaker management (one channel).

\subsection{The junction box slow control electronics}

Output breaker manipulation and measurement of currents, temperatures and humidity are the main activities of the triply redundant JB slow control system. This system uses eight fibres in the undersea cable and is based on three control cards built in two different technologies.

In the first of these, two identical cards ('A' \& ' $\mathrm{B}$ ') communicate with the shore station through $160 \mathrm{Mb} / \mathrm{G}$-links, using the Agilent HDMP-1022/1024 transmitter/ receiver chip set with the Photon Techno PT5543-13-3-SC laser emitter and PT6143-155-SC receiver operating at $1550 \mathrm{~nm}$. Each card can simultaneously stream 16-bit digitized data from 48

5"MACC plus" Zero flux current transformer, 10A full scale, $100 \mu \mathrm{A}$ resolution: mfr Hitec bv, 7555 CS Hengelo, Netherlands:. 
internal JB temperature and humidity sensors, and 24bit data at $2.6 \mathrm{kHz}$ sampling from a group of 4 "MACC plus" inductive current sensors. This latter data is passed to a shore-based Texas Instruments TMS320C5510 200MHz digital signal processor (DSP) and used for sinusoid reconstruction and rms current calculation.

A third card (' $\mathrm{C}$ '), powered by lithium batteries, is based on a T.I. MSP430F149 microcontroller with $60 \mathrm{~Kb}$ flash and 2048b RAM memory, equipped with eight 12 bitADC entries and 45 digital I/O ports. This card communicates at 1200 Baud using an NDL7701 laser uplink operating at 1550nm and an LPD80 pin diode receiver. This channel has $5 \mu \mathrm{A}$ consumption in sleep mode and a maximum of $100 \mathrm{~mA}$ for short periods, when the up-link is transmitting.

\subsection{Fibreoptic signal distribution in the junction box}

Each JB output connector contains four optical fibres with the following functions;

- $\quad$ DAQ $\mathrm{Rx}_{\mathrm{n}}$ (downlink from shore; $\mathrm{n}=1 \rightarrow 16$ );

- $\quad$ DAQ $x_{n}$ (data up-link to shore; $\mathrm{n}=1 \rightarrow 16$ );

- Clock channel A;

- Clock channel B;

DAQ Tx and Rx are specific to each line, and are accommodated using 32 fibres in the undersea cable, which are point-to-point spliced in the JB hub to their respective fibres in the 16 output connectors.

The distributed clock signal, vital for time referencing photomultiplier data to nanosecond precision, is transmitted with 4-fold redundancy. Pulse trains from two independent, identical clock transmitters at the shore station are split for broadcast on four undersea fibres. In the JB they are routed via dual-input 16-way passive splitters so that clock pulse trains from either or both transmitters are available on every output connector.

All internal fibre connections are made by fusion splicing, giving a loss of around $0.01 \mathrm{~dB}$ per joint.

\section{The Graphical user interface}

A Java-based graphical user interface has been developed for monitor and control of the power delivery system and underwater JB. Sensor data are converted into engineering units (e.u.) for on-screen presentation and are written at regular intervals into a data base.

The data base also stores e.u. warning and alarm thresholds for each sensor, together with the priority (response) level of each threshold. Low priority alarms alert the operator with on-screen messages while higher priorities can also generate SMS text messages and, depending on the criticality of the sensor, may trigger a power shutdown after a predetermined delay.

\section{Operational results and conclusion}

The power delivery and junction box slow control system has been in operation since deployment in December 2002 and has been used to power a variety of prototype detection lines and instrumentation packages. Increased energy demands will be soon be made of it when the first full length detection lines are deployed in early 2006. The full power compliment of 12 lines is expected to be operational by late 2007 .

Future extensions to the DSP system are foreseen to detect leakage current in the $41 \mathrm{~km}$ undersea cable. Sensitivities of the order of $250 \mu \mathrm{A}$ [6] are expected, giving an early indication of water infiltration so that a prompt repair of any damage may be effected.

\section{References}

[1]ANTARES Technical Design Report version 1.0 July 2, 2001 via http://antares.in2p3.fr

[2] G. Hallewell, Nucl. Instr. Meth. A502 (2003) 138.

[3]G.Hallewell, "Technological Developments for Deep Underwater Neutrino Telescopes”, ICFA Instrumentation. Bulletin. 25:17-38, 2002.

[4]A. Calzas, ANTARES Internal note 3-ENE-01-13-B; via http://antares.in2p3.fr

[5]M. Anghinolfi et al, The underwater power and communications hub of the ANTARES neutrino telescope

To be published in Proc 2005 IEEE Nuclear Science Symposium, Puerto Rico, November 2005

[6] J. Carr, G. Hallewell and P. Vernin, ANTARES internal note 3ENE-01-21-A via http://antares.in2p3.fr 